\title{
High-Throughput and Sensitive Assay for Amphotericin B Interaction with Lipid Membrane on the Model Membrane Systems by Surface Plasmon Resonance
}

\author{
Masako Onishi* and Hiroshi Kamimori \\ Pharmaceutical Research Center, Shionogi \& Co., Ltd.; 3-1-1 Futaba-cho, Toyonaka, Osaka 561-0825, Japan.
} Received November 26, 2012; accepted January 8, 2013

In the present study, we developed a high-throughput and sensitive assay for interactions of amphotericin B (AmB) with two model lipid membranes, which mimicked mammal cell membrane and fungal membrane using surface plasmon resonance (SPR). The binding kinetics of AmB to the membrane could be analyzed by multiple sensorgrams obtained at different AmB concentrations, indicating that the binding properties could be clarified for an approximately 7-fold concentration range. AmB showed an approximately 18-fold higher affinity for ergosterol-containing membrane than for cholesterol-containing membrane. We also optimized the procedure for the reproducible immobilization of liposome containing the sterols and the estimation of binding kinetics of AmB to the lipid membranes, and the sensitivity of AmB to membrane interaction was 20 -fold higher, compared with the reported method. The throughput of the established method for the binding kinetics characterization was calculated to be 10 compounds a day. The results demonstrate that the established SPR method could be a valuable tool for predicting selective binding to sterol-containing membranes.

Key words amphotericin B; surface plasmon resonance; lipid membrane

Amphotericin B (AmB), an antifungal polyene macrolide antibiotic produced by Streptomyces nodosus, is known as the gold-standard drug for the treatment of many systemic fungal infections or "deep mycoses" that could be life-threatening for specific populations, such as immunocompromised, transplant and cancer patients. ${ }^{1)}$ The route of administration for antifungal therapies with $\mathrm{AmB}$ is intravenous (i.v.) infusion. This treatment requires hospitalization and has numerous side effects, such as nephrotoxic potential. ${ }^{2-4)}$ Therefore, the development of new antifungal antibiotics is desired for more simplified treatment and reduction of side effects.

AmB has a highly hydrophobic, water-insoluble molecule with amphiphilic properties, and the drug product formulation of AmB is quite challenging. ${ }^{5)}$ Although there are various ways to formulate new drug products based on the AmB skeleton, no formulation of $\mathrm{AmB}$ is currently available for systemic treatment of infection after oral administration. It is generally accepted that an ion-permeable channel formed across the bilayer membrane is responsible for the biological activity of AmB. ${ }^{6,7)}$ The antibiotic activity of $\mathrm{AmB}$ is believed to be primarily due to a higher affinity for ergosterol, a primary fungal sterol, than for cholesterol in mammalian membranes. ${ }^{8-11)}$ Thus, in order to develop a new drug based on the AmB skeleton with better antibiotic efficacy and fewer side effects, such as nephrotoxicity, diverse drug candidates need to be examined for their membrane affinity.

The affinity of AmB for lipid membranes is one of the critical factors influencing selectivity and potency. Surface plasmon resonance (SPR)-based biosensors have been already applied to study the membrane-binding properties of drugs with the aim of gaining insight into their mechanism of action. ${ }^{12,13)}$ We and our collaborators have already established a method to evaluate the interactions of various peptides, including antimicrobial peptides, to the lipid membranes using a SPR biosensor, Biacore, and demonstrated that the kinetic rate constants and the affinity of membrane interactions can be readily determined, which in turn provides important insights into the mechanism of peptide-membrane interactions. ${ }^{14-19)}$

In the present study, we developed a high-throughput and sensitive assay of interactions of AmB with two model lipid membranes, which mimic mammal cell membrane and fungal membrane, using our established SPR technique, focusing on the analysis of the binding kinetics of lipid membranes. ${ }^{14-19)}$ Our ultimate target of the related study is to establish a valuable tool for predicting the selective binding and antimycotic activity of antifungals with the SPR system, including the toxicity properties of the target compounds.

The sterol-containing lipid membrane of binding properties of AmB by SPR was previously reported by Mouri et al., ${ }^{20)}$ and the method involved the kinetic data obtained from one AmB sensorgram, and the selectivity of AmB to a sterol-containing lipid membrane would be shown by SPR. There would be a few progressive points by our SPR technique, including the throughput and sensitivity and binding kinetic analysis with multiple sensorgrams.

This paper describes our high-throughput and sensitive assay of the binding properties of AmB-lipid membranes using the SPR system Biacore S51, ${ }^{21-23)}$ and the binding characteristics of $\mathrm{AmB}$ to two model lipid membrane analyzed by the binding kinetics using the global fitting of multiple sensorgrams over an approximately 7-fold range of AmB concentrations. Also the present assay showed satisfactory reproducibility of the immobilization of the model phospholipid membranes onto the sensor chip. The estimation of binding kinetics of AmB to the lipid membranes is discussed. Further the binding properties of $\mathrm{AmB}$ to model lipid membranes around the plasma concentration of the treatment range in vivo are discussed. 


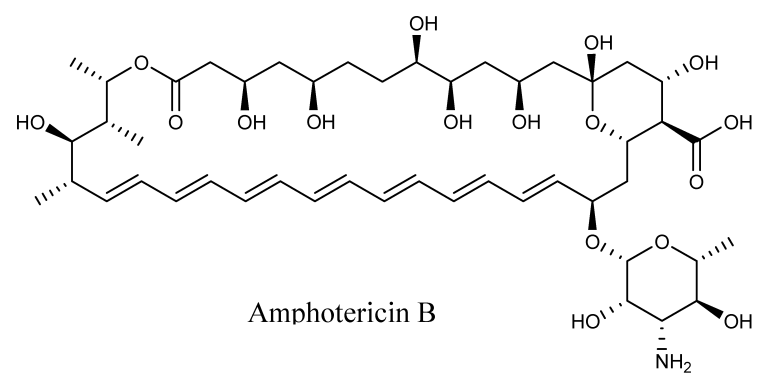

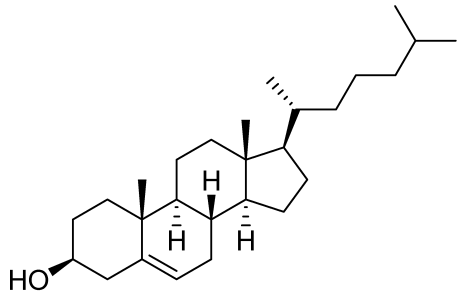

Cholesterol

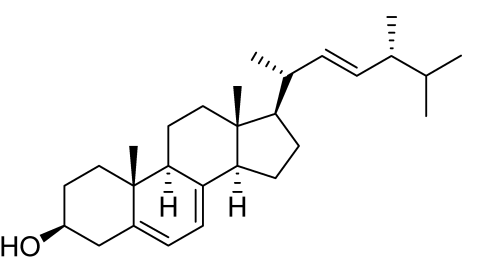

Ergosterol

Fig. 1. Chemical Structures of AmB, Cholesterol, and Ergosterol

\section{MATERIALS AND METHODS}

Chemicals and Reagents Amphotericin B, dimethyl sulfoxide (DMSO), ergosterol, and cholesterol were purchased from Sigma-Aldrich Inc. (St. Louis, MO, U.S.A.). 1-Palmitoyl2-oleoyl-sn-glycero-3-phosphocholine (POPC) was purchased from Avanti Polar Lipids, Inc. (Alabaster, AL, U.S.A.). 3-[(3-Cholamidpropyl)-dimethylammonio]propanesulfonate (CHAPS) and chloroform were purchased from Wako Pure Chemical Industries, Ltd. (Osaka, Japan). Water was purchased from Nacalai Tesque, Inc. (Kyoto, Japan). Concentrated phosphate bufferd saline (PBS) was purchased from GE Healthcare U.K. Ltd. (Buckinghamshire, England). The chemical structures of AmB, ergosterol, and cholesterol are listed in Fig. 1.

Apparatus The SPR system used was a BIACORE S51 analytical system and Series S Sensor Chip L1 used for immobilization of liposomes (GE Healthcare U.K. Ltd., Buckinghamshire, England). The running buffers used were PBS containing 10\% DMSO for immobilization of lipid membranes on the chip and the estimation of interaction of the AmB to the membrane. The washing solution was 50\% DMSO and the regeneration solution was $20 \mathrm{mmol} / \mathrm{L}$ CHAPS. All solutions were freshly prepared, degassed and filtered through a $0.22 \mu \mathrm{m}$ filter.

Liposome Preparation In order to prepare individual stock solutions, dry POPC, ergosterol and cholesterol were dissolved in chloroform. These stock solutions were prepared at the desired ratios: POPC-ergosterol $(4: 1, \mathrm{v} / \mathrm{v})$ and POPCcholesterol $(4: 1, \mathrm{v} / \mathrm{v})$. The solvents were evaporated under a stream of nitrogen, and the lipids were dried under centrifuged evaporation. The lipids were resuspended in PBS. The resultant lipid dispersion (a concentration of $4 \mathrm{mmol} / \mathrm{L}$ POPCergosterol $(4: 1, \mathrm{v} / \mathrm{v})$ and POPC-cholesterol $(4: 1, \mathrm{v} / \mathrm{v}))$ was sonicated until nearly clear and extruded 21 times through polycarbonate filters to obtain small unilamellar vesicles (SUVs) of $100 \mathrm{~nm}$ size (LiposoFast, pore diameter $100 \mathrm{~nm}$ ). The extruded lipid dispersion was prepared at 8-fold dilution in PBS containing 10\% DMSO (a concentration of $0.5 \mathrm{mmol} / \mathrm{L}$ with respect to phospholipid for POPC-ergosterol $(4: 1, \mathrm{v} / \mathrm{v})$ and POPC-cholesterol $(4: 1, \mathrm{v} / \mathrm{v}))$.

Formation of Lipid Bilayer Membranes The L1 sensor chip surface was washed with three injections of $15 \mu \mathrm{L}$ of $20 \mathrm{mmol} / \mathrm{L}$ CHAPS at a flow rate of $30 \mu \mathrm{L} / \mathrm{min}$. SUVs were immediately applied to the sensor chip at a flow rate of $10 \mu \mathrm{L} /$ min for liposome capture for $500 \mathrm{~s}$.

AmB Binding to the Bilayer Membrane AmB assay solutions were prepared by dissolving in PBS containing $10 \%$ DMSO from 0.25 to $20 \mu \mathrm{mol} / \mathrm{L}$. The AmB solution $(100 \mu \mathrm{L}$, $200 \mathrm{~s}$ ) was injected over the lipid surface at a flow rate of $30 \mu \mathrm{L} / \mathrm{min}$ to avoid limitation by mass transfer. On completion of injection, the buffer flow was continued to allow a dissociation time of $400 \mathrm{~s}$. All binding experiments were carried out at $25^{\circ} \mathrm{C}$. The affinity of $\mathrm{AmB}$ for the lipid bilayer membrane was determined from analysis of a series of response curves collected at six different AmB concentrations injected over each lipid surface in triplicate. After injection of the AmB assay solution, $15 \mu \mathrm{L}$ of $20 \mathrm{mmol} / \mathrm{L}$ CHAPS was injected at a flow rate of $30 \mu \mathrm{L} / \mathrm{min}$ to remove any captured liposome and AmB from the L1 sensor chip surface. This procedure indicated that the liposome capture was freshly performed for each assay cycle, resulting in a sensor chip surface that could be recoated with fresh liposome containing the sterol for the next binding cycle.

Data Analysis The sensorgrams for each AmB-lipid interaction were analyzed by curve fitting using numerical integration analysis $(1.5-10 \mu \mathrm{mol} / \mathrm{L})$. The data were fitted globally by simultaneously fitting the AmB sensorgrams obtained at six different concentrations using BIA evaluation software (Ver. 4.1). The two-state reaction model was applied to the resultant $\mathrm{AmB}$ binding curves to estimate the association and dissociation rate constants and this model was chosen on the basis of previous studies of AmB-membrane interactions. ${ }^{14-19)}$ This model describes two steps, which in terms of AmB-lipid interaction may correspond to:

$$
\mathrm{A}+\mathrm{L} \underset{k_{\mathrm{d} 1}}{\stackrel{k_{\mathrm{a} 1}}{\longrightarrow}} \mathrm{AL} \underset{k_{\mathrm{d} 2}}{\stackrel{k_{\mathrm{a} 2}}{\longrightarrow}} \mathrm{AL} *
$$

where $\mathrm{A}$ is the $\mathrm{AmB}, \mathrm{L}$ is the lipid membrane, $\mathrm{AL}$ is the 
initial complex formed by the lipid membrane and AmB via, for example, electrostatic interaction, and the complex $\mathrm{AL}$ changes to $\mathrm{AL}^{*}$, which cannot dissociate directly to $\mathrm{A}+\mathrm{L}$ and which may correspond to the insertion of the AmB into the lipid bilayer.

The corresponding differential rate equations for this reaction model are represented by

$$
\begin{aligned}
& \mathrm{d} R_{1} / \mathrm{d} t=k_{\mathrm{a} 1} \cdot C_{\mathrm{A}}\left(R_{\max }-R_{1}-R_{2}\right)-k_{\mathrm{d} 1} \cdot R_{1}-k_{\mathrm{a} 2} \cdot R_{1}+k_{\mathrm{d} 2} \cdot R_{2} \\
& \mathrm{~d} R_{2} / \mathrm{d} t=k_{\mathrm{a} 2} \cdot R_{1}-k_{\mathrm{d} 2} \cdot R_{2}
\end{aligned}
$$

\section{RESULTS AND DISCUSSION}

Optimization of Immobilization of Model Lipid Membranes on the L1 Sensor Chip In previous studies, ${ }^{20)}$ the methodology using a modified CM5 sensor chip for the assay of the binding of $\mathrm{AmB}$ and the sterol-containing lipid membrane was reported using SPR, and the selectivity of AmB for sterol-containing lipid membrane was shown, indicating that AmB has a higher affinity for ergosterol membrane than cholesterol membrane. In this SPR study, two types of liposomes containing the sterol were used as model lipid membranes. POPC-cholesterol $(4: 1, \mathrm{v} / \mathrm{v})$ was used to mimic of mammalian membranes, while POPC-ergosterol (4:1, v/v) was used as a model fungal membrane. ${ }^{20)}$ We aimed to establish a better assay of AmB-lipid membrane interaction with our SPR system, considering the time-consuming process for sensor chip modification, the damage to the immobilized liposome on the chip due to repeated and consecutive AmB injection, the carryover between each binding assay, and the throughput and sensitivity. In other words, we tried to establish a high-throughput, sensitive, and reproducible binding assay of AmB-sterol containing lipid membrane using an L1 sensor chip, which is usually used for lipid-membrane systems. ${ }^{14-19)}$

First, in order to prevent the carryover of AmB between each binding assay of AmB-lipid membranes, we investigated the regeneration procedure of the L1 chip for each assay because the AmB retained in the liposome on the sensor chip would affect the exact estimation of binding kinetics. As the procedure of regeneration of sensor chip surface has been also optimized, the sensor chip surface could be completely regenerated to the original surface by injecting $20 \mathrm{mmol} / \mathrm{L}$ CHAPS

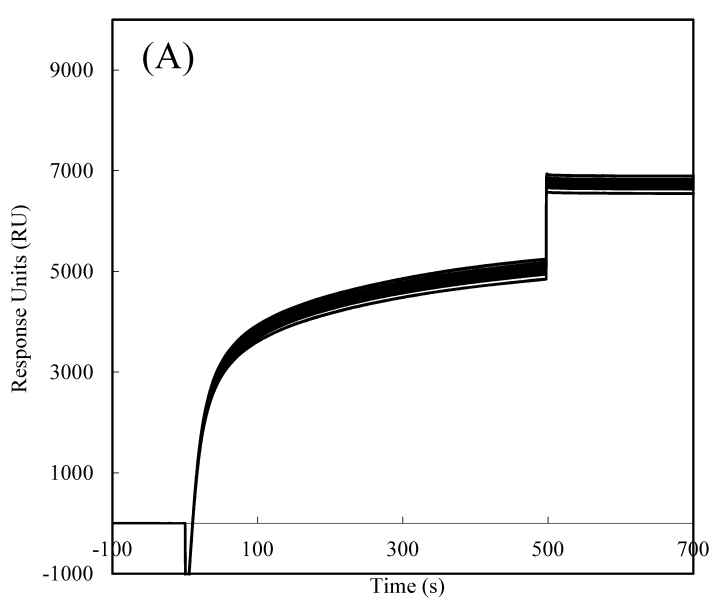

for each assay. The desirable regeneration of the surface chip could be judged by the return of the response to the original baseline. This procedure indicated that the sensor chip surface had been completely recoated with fresh liposome containing the sterol for the next binding cycle. On the other hand, the regeneration, in which $\mathrm{AmB}$ was only removed from the immobilized liposome on the sensor chip, was no effective in this assay because it was very difficult to find an appropriate regeneration solution. Under the optimized regeneration procedure, the response units (RU) of each lipid membrane immobilized on the L1 chip was measured in separate experiments $(n=24)$. The average RU, standard deviation and relative standard deviation for each lipid type are listed in Table 1 and the sensorgrams are presented in Fig. 2. In Fig. 2, the stabilized baseline of the sensorgram captured liposome (from 500 to 700 s) for calculation of binding level of liposomes rose due to the bulk effect, compared with that of the completion of liposome captured on the sensor chip (at 500s). The reason for this is due to the concentration of DMSO for the running buffer was higher than that of injected liposome solution. The approximate RUs for POPC-ergosterol (4:1, v/v) and POPCcholesterol $(4: 1, \mathrm{v} / \mathrm{v})$ were 6800 and 5600 , respectively. The relative standard deviations in RU for the two lipid membranes were calculated to be less than $1.3 \%$. The results demonstrated a high level of reproducibility in the immobilization of lipid membranes on the sensor chip. Also, the approximately 6000 RU of lipid membranes provides reproducible sensorgrams and binding kinetics analysis for drug-membrane interaction, especially, by two-state reaction model analysis. ${ }^{12,16)}$

Interaction of AmB with Lipid Membranes Figure 3 shows representative sensorgrams of the binding of AmB to the immobilized POPC-ergosterol $(4: 1, \mathrm{v} / \mathrm{v})$ and

Table 1. Response Units and Reproducibility of Immobilization of Lipid Membranes on L1 Chip

\begin{tabular}{cccc}
\hline \hline \multirow{2}{*}{ Lipid type } & \multicolumn{2}{c}{ Response units } & \\
\cline { 2 - 3 } & Mean $(n=24)$ & S.D. $^{a}{ }^{a}$ & \\
\hline POPC-ergosterol $(4: 1)$ & 5622 & 29 & 0.5 \\
POPC-cholesterol $(4: 1)$ & 6759 & 96 & 1.4
\end{tabular}

a) Standard deviation. $b$ ) Relative standard deviation.

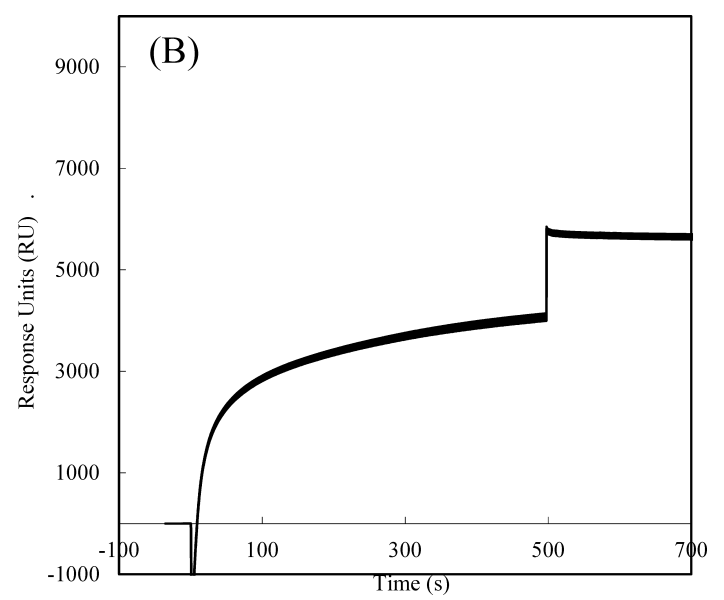

Fig. 2. Reproducibility of Sensorgrams for Immobilization of POPC-Cholesterol (4:1, v/v) (A) and POPC-Ergosterol (4:1, v/v) (B) on the L1 Sensor Chip Surface 
POPC-cholesterol $(4: 1, \mathrm{v} / \mathrm{v})$ on the chip. In these cases, there was a proportional increase in the response with increases in $\mathrm{AmB}$ concentration, indicating that the system had not reached saturation. However, the response at more than $20 \mu \mathrm{mol} / \mathrm{L}$ of AmB could not be increased proportionally with increases in concentration. In addition, comparison of the SPR response for $\mathrm{AmB}$ revealed that a higher proportion binds to POPC-ergosterol $(4: 1, \mathrm{v} / \mathrm{v})$ than to POPC-cholesterol $(4: 1$, $\mathrm{v} / \mathrm{v})$, especially in the dissociation phase.

The association $\left(k_{\mathrm{a} 1}, k_{\mathrm{a} 2}\right)$ and dissociation $\left(k_{\mathrm{d} 1}, k_{\mathrm{d} 2}\right)$ constants and the affinity constant $\left(K_{1}, K_{2}\right.$, and $\left.K\right)$ estimated by the twostate reaction model are listed in Table 2 . The reproducibility of each parameter by repeated assay was good. This model was selected on the basis of the multistep model of AmBlipid or peptide-lipid interactions, which may involve initial binding interaction between the $\mathrm{AmB}$ and the head group of liposome (step 1, characterized by $k_{\mathrm{a} 1}, k_{\mathrm{d} 1}$ ), including the electrostatic interaction, and followed by reorientation and/or insertion of the analyte into the hydrophobic interior (step 2, characterized by $k_{\mathrm{a} 2}, k_{\mathrm{d} 2}$ ) as previously described. ${ }^{14-20)}$ In this model, the values of $k_{\mathrm{a} 1} / k_{\mathrm{d} 1}\left(K_{1}\right)$ and $k_{\mathrm{a} 2} / k_{\mathrm{d} 2}\left(K_{2}\right)$ correspond to the affinity constants for initial binding interaction (including the electrostatic interaction) and hydrophobic interaction between AmB and lipid, respectively.

The results of the kinetic analysis repeatedly showed an approximately 18-fold higher affinity constant of $\mathrm{AmB}$ for POPC-ergosterol $(4: 1, \mathrm{v} / \mathrm{v})$ than POPC-cholesterol $(4: 1$, $\mathrm{v} / \mathrm{v})$. The affinity of the first step of interaction of $\mathrm{AmB}$ to the head group of liposome was similar because only a 2 fold difference of $K_{1}$ values was observed between the two
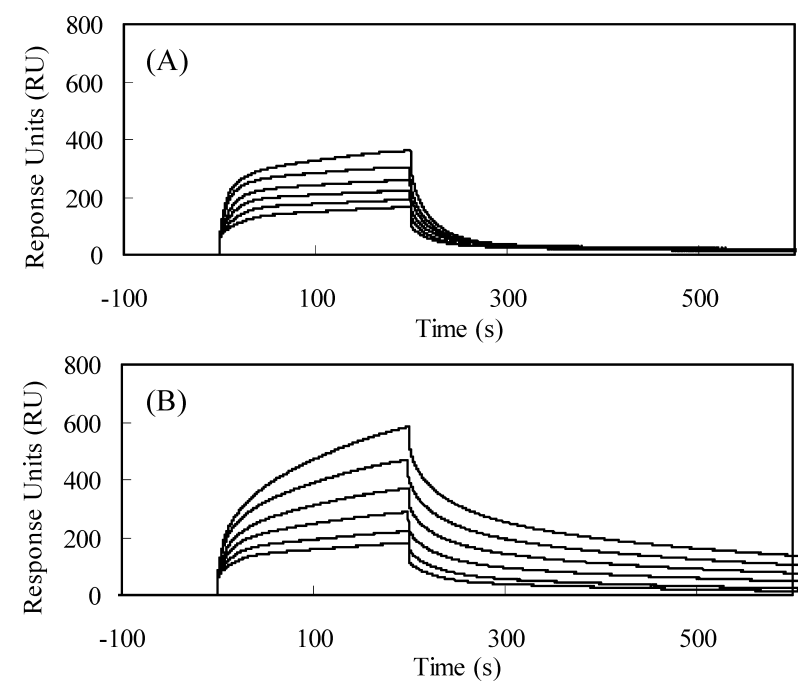

Fig. 3. Sensorgrams for AmB Binding to POPC-Cholesterol $(4: 1, \mathrm{v} / \mathrm{v})$ (A) and POPC-Ergosterol $(4: 1, \mathrm{v} / \mathrm{v})$ (B) Liposomes Immobilized on the L1 Sensor Chip Surface

AmB concentration $=1.5-10 \mu \mathrm{mol} / \mathrm{L}$. membranes. On the other hand, the values of the hydrophobic association rate $\left(k_{\mathrm{a} 2}\right)$ in the second step were significantly different between the two membranes, indicating that the force of hydrophobic insertion of AmB to the cholesterol-containing membrane was approximately 70 -fold stronger than that of the ergosterol-containing membrane. In addition, the value of the dissociation rate $\left(k_{\mathrm{d} 2}\right)$ of $\mathrm{AmB}$ to the ergosterol-containing membrane in the second step was lower by more than 1000 fold than that of cholesterol-containing membrane. The results indicated that $\mathrm{AmB}$ could dissociate extremely more slowly from the ergosterol-containing membrane than the cholesterolcontaining membrane. This slow dissociation could be clearly observed from the sensorgrams (Fig. 3), and suggesting that the higher affinity to ergosterol-containing membrane was mainly due to the slower dissociation of AmB from the formation of an AmB ion channel in the hydrophobic membrane interior. $^{24,25)}$

AmB is well-known as an antifungal drug, which is administered at doses close to those producing side effects. ${ }^{26}$ ) In order to prevent the side effects, dose adjustment at the clinical site should be usually considered to provide the effective treatment. For example, side effects, such as nephrotoxicity, would be caused by cytoclasis due to AmB binding to a cholesterol-containing membrane. ${ }^{27,28)}$ From the viewpoint of AmB having these characteristics as an antifungal drug, it might be speculated that the dramatically difference of affinity between ergosterol- and cholesterol-containing membrane would not be observed, indicating that $\mathrm{AmB}$ might show also the binding affinity to cholesterol-containing membrane.

We would propose that the established method using an SPR system might provide the capability to estimate the balance of beneficial effect and toxic potential for new drug design in the future. As the approximately 18-fold selectivity to ergosterolcontaining membrane in the case of $\mathrm{AmB}$ was observed in the SPR system, the ideally desirable antifungal drug, which has a high beneficial effect and low toxic potential, might possess higher selectivity for the ergosterol-containing membrane in the SPR system.

In trying to establish an effective and efficient method for characterizing the binding kinetics between liposome membranes and drugs, we estimate that the throughput of this method is 10 compounds a day, thus making high throughput SPR analysis possible when compared with other reported methods. Also the method can be easily applied to estimate the various drug membrane interactions by high-throughput analysis in vitro.

Binding Properties around the Plasma Concentration of the Treatment Range We confirmed the sensorgrams of the binding of $\mathrm{AmB}$ to the immobilized POPC-ergosterol (4:1, $\mathrm{v} / \mathrm{v}$ ) from 0.25 to $1.5 \mu \mathrm{mol} / \mathrm{L}$ (Fig. 4). The AmB solution was injected over the lipid surface at a flow rate $30 \mu \mathrm{L} / \mathrm{min}$ for $50 \mathrm{~s}$. On completion of injection, the buffer flow was continued

Table 2. Kinetic and Affinity Constants for Interaction between AmB (1.5-10 $\mu \mathrm{mol} / \mathrm{L})$ and Sterol-Containing POPC Liposomes Obtained from the TwoState Reaction Model $^{a)}$

\begin{tabular}{cccccccc}
\hline \hline Lipid type & $k_{\mathrm{a} 1}(1 / \mathrm{Ms})^{a)}$ & $k_{\mathrm{d} 1}\left(\times 10^{-3}, 1 / \mathrm{s}\right)^{a)}$ & $k_{\mathrm{a} 2}\left(\times 10^{-4}, 1 / \mathrm{s}\right)^{a)}$ & $k_{\mathrm{d} 2}\left(\times 10^{-5}, 1 / \mathrm{s}\right)^{a)}$ & $K_{1}\left(\times 10^{5}, 1 / \mathrm{M}\right)^{a)}$ & $K_{2}^{a)}$ & $K\left(\times 10^{5}, 1 / \mathrm{M}\right)^{a)}$ \\
\hline POPC-ergosterol $^{b)}$ & $1600 \pm 430^{d)}$ & $7.52 \pm 1.85$ & $3.96 \pm 2.41$ & $3.44 \pm 2.00$ & $2.12 \pm 0.34$ & $12.8 \pm 3.7$ & $28.5 \pm 4.9$ \\
POPC-cholesterol $^{c)}$ & $9390 \pm 400$ & $86.6 \pm 4.7$ & $130 \pm 9$ & $1800 \pm 200$ & $1.09 \pm 0.09$ & $0.724 \pm 0.091$ & $1.87 \pm 0.06$ \\
\hline
\end{tabular}

a) The association rate constants $\left(k_{\mathrm{a} 1}\right.$ and $\left.k_{\mathrm{a} 2}\right)$, the dissociation rate constants $\left(k_{\mathrm{d} 1}\right.$ and $\left.k_{\mathrm{d} 2}\right)$, and the equilibration constants $K_{1}\left(k_{\mathrm{a} 1} / k_{\mathrm{d} 1}\right), K_{2}\left(k_{\mathrm{a} 2} / k_{\mathrm{d} 2}\right)$ and $K\left(K_{1}+K_{1} K_{2}\right)$. b) POPC-ergosterol $(4: 1, \mathrm{v} / \mathrm{v}) . c)$ POPC-cholesterol $(4: 1, \mathrm{v} / \mathrm{v}) . d)$ Mean \pm standard deviation $(n=3)$. 


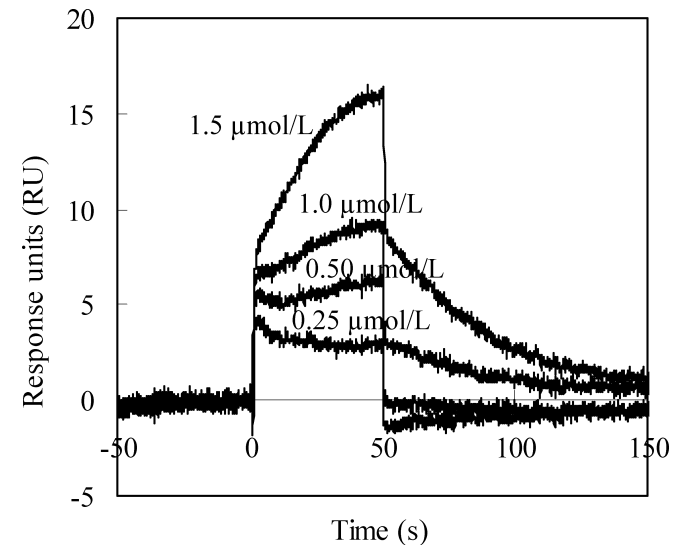

Fig. 4. Sensorgrams for AmB Binding to POPC-Ergosterol (4:1, v/v) Liposomes Immobilized on the L1 Sensor Chip Surface

AmB concentration $=0.25-1.5 \mu \mathrm{mol} / \mathrm{L}$

to allow a dissociation time of $100 \mathrm{~s}$. The $\mathrm{AmB}$ at 0.25 to $0.5 \mu \mathrm{mol} / \mathrm{L}$ was observed only to bind to the head group of the liposome membrane (such as, "Langmuir binding model"), judging from the shapes of the sensorgrams. ${ }^{29)}$ In this concentration range, the insertion of $\mathrm{AmB}$ to the hydrophobic interior of the ergosterol membrane would not have occurred yet. On the other hand, AmB at from 1.0 to $1.5 \mu \mathrm{mol} / \mathrm{L}$ would be observed to initiate to appear slightly the hydrophobic interaction between $\mathrm{AmB}$ and ergosterol membrane, judging from the shapes of the sensorgrams, especially, in the dissociation phase.

As the effective concentration of $\mathrm{AmB}$ in human plasma for therapy is known to be over $1.0 \mu \mathrm{mol} / \mathrm{L},{ }^{30)}$ the interaction of $\mathrm{AmB}$ with a membrane at over $1.0 \mu \mathrm{mol} / \mathrm{L}$ would be related to the beneficial effects of $\mathrm{AmB}$ as noted previously. That is to say, the entering of $\mathrm{AmB}$ into the hydrophobic interior would be related to the initiation of beneficial effects. This is the first report of an actual demonstration of the relationship between the shapes of sensorgrams and the effectivity of drugs for therapy.

In this SPR system, the membrane binding down to $0.25 \mu \mathrm{mol} / \mathrm{L}$ of $\mathrm{AmB}$ could be detected, revealing that the sensitivity of AmB to membrane interaction was 20-fold higher, compared with that reported previously. ${ }^{20)}$

\section{CONCLUSION}

We developed a high-throughput and sensitive assay for the interaction of $\mathrm{AmB}$ with lipid membrane. The present assay showed satisfactory reproducibility of immobilization of the model phospholipid membranes onto the sensor chip and the estimation of binding kinetics of $\mathrm{AmB}$ to the lipid membranes, indicating that the method could be used to estimate the selective binding of $\mathrm{AmB}$ to model mammalian or fungal phospholipid membranes. We also demonstrated that the affinity of $\mathrm{AmB}$ for the ergosterol-containing lipid membrane was approximately 18 -fold higher than that for cholesterol-containing lipid membrane. This higher affinity would be especially due to the slower dissociation of $\mathrm{AmB}$ from the ergosterol membrane than from the cholesterol membrane. Also, the binding properties of $\mathrm{AmB}$ to model lipid membranes around the plasma concentration of treatment range in vivo could be basically explained by the change of sensorgrams. The results demonstrate that the established SPR method can be a valuable tool for predicting the selective binding and antimycotic activity of antifungals.

Acknowledgment Special acknowledgment to Dr. Michio Murata from Osaka University to his support.

\section{REFERENCES}

1) Vartivarian SE, Anaissie EJ, Bodey GP. Emerging fungal pathogens in immunocompromised patients: classification, diagnosis, and management. Clin. Infect. Dis., 17 (Suppl. 2), S487-S491 (1993).

2) Kaloyanides GJ. Antibiotic-related nephrotoxicity. Nephrol. Dial. Transplant., 9 (Suppl. 4), 130-134 (1994).

3) Sabra R, Branch RA. Amphotericin B nephrotoxicity. Drug Saf., 5, 94-108 (1990).

4) Sawaya BP, Briggs JP, Schnermann J. Amphotericin B nephrotoxicity: the adverse consequences of altered membrane properties. $J$. Am. Soc. Nephrol., 6, 154-164 (1995).

5) Segarra I, Movshin DA, Zarif L. Pharmacokinetics and tissue distribution after intravenous administration of a single dose of amphotericin B cochleates, a new lipid-based delivery system. J. Pharm. Sci., 91, 1827-1837 (2002).

6) Bolard J. How do the polyene macrolide antibiotics affect the cellular membrane properties? Biochim. Biophys. Acta, 864, 257-304 (1986).

7) Hartsel SC, Bolard J. Amphotericin B: New life for an old drug. Trends Pharmacol. Sci., 17, 445-449 (1996).

8) Readio JD, Bittman R. Equilibrium binding of amphotericin B and its methyl ester and borate complex to sterols. Biochim. Biophys. Acta, 685, 219-224 (1982).

9) Vertut-Croquin A, Bolard J, Chabbert M, Gary-Bobo C. Differences in the interaction of the polyene antibiotic amphotericin $\mathrm{B}$ with cholesterol- or ergosterol-containing phospholipid vesicles. A circular dichroism and permeability study. Biochemistry, 22, 2939-2944 (1983).

10) Milhaud J, Hartmann MA, Bolard J. Interaction of the polyene antibiotic amphotericin B with model membranes: differences between small and large unilamellar vesicles. Biochimie, 71, 49-56 (1989).

11) Vertut-Croquin A, Bolard J, Gary-Bobo CM. Enhancement of amphotericin B selectivity by antibiotic incorporation into gel state vesicles. A circular dichroism and permeability study. Biochem. Biophys. Res. Commun., 125, 360-366 (1984).

12) Baird CL, Courtenay ES, Myszka DG. Surface plasmon resonance characterization of drug/liposome interactions. Anal. Biochem., 310, 93-99 (2002).

13) Abdiche YN, Myszka DG. Probing the mechanism of drug/lipid membrane interactions using Biacore. Anal. Biochem., 328, 233-243 (2004).

14) Mozsolits H, Unabia S, Ahmad A, Morton CJ, Thomas WG, Aguilar MI. Electrostatic and hydrophobic forces tether the proximal region of the angiotensin II receptor (AT1A) carboxyl terminus to anionic lipids. Biochemistry, 41, 7830-7840 (2002).

15) Mozsolits H, Lee TH, Clayton AH, Sawyer WH, Aguilar MI. The membrane-binding properties of a class A amphipathic peptide. Eur. Biophys. J., 33, 98-108 (2004).

16) Kamimori H, Hall K, Craik DJ, Aguilar MI. Studies on the membrane interactions of the cyclotides kalata B1 and kalata B6 on model membrane systems by surface plasmon resonance. Anal. Biochem., 337, 149-153 (2005).

17) Kamimori H, Blazyk J, Aguilar MI. Lipid membrane-binding properties of tryptophan analogues of linear amphipathic $\beta$-sheet cationic antimicrobial peptides using surface plasmon resonance. Biol. Pharm. Bull., 28, 148-150 (2005).

18) Kamimori H, Unabia S, Thomas WG, Aguilar MI. Evaluation of the membrane-binding properties of the proximal region of the 
angiotensin II receptor (AT1A) carboxyl terminus by surface plasmon resonance. Anal. Sci., 21, 171-174 (2005).

19) Lerch M, Kamimori H, Folkers G, Aguilar MI, Beck-Sickinger AG, Zerbe O. Strongly altered receptor binding properties in PP and NPY chimeras are accompanied by changes in structure and membrane binding. Biochemistry, 44, 9255-9264 (2005).

20) Mouri R, Konoki K, Matsumori N, Oishi T, Murata M. Complex formation of amphotericin B in sterol-containing membranes as evidenced by surface plasmon resonance. Biochemistry, 47, 7807-7815 (2008).

21) Myszka DG. Analysis of small-molecule interactions using Biacore S51 technology. Anal. Biochem., 329, 316-323 (2004).

22) Frostell-Karlsson $\AA \mathrm{SA}$, Widegren $\mathrm{H}$, Green $\mathrm{CE}$, Hämäläinen $\mathrm{MD}$, Westerlund L, Karlsson R, Fenner $\mathrm{K}$, van de Waterbeemd $\mathrm{H}$. Biosensor analysis of the interaction between drug compounds and liposomes of different properties; a two-dimensional characterization tool for estimation of membrane absorption. J. Pharm. Sci., 94, 25-37 (2005).

23) Cimitan S, Lindgren MT, Bertucci C, Danielson UH. Early absorption and distribution analysis of antitumor and anti-AIDS drugs: lipid membrane and plasma protein interactions. J. Med. Chem., 48, 3536-3546 (2005).

24) de Kruijff B, Demel RA. Polyene antibiotic-sterol interactions in membranes of Acholeplasma laidlawii cells and lecithin liposomes.
III. Molecular structure of the polyene antibiotic-cholesterol complexes. Biochim. Biophys. Acta, 339, 57-70 (1974).

25) Baginski M, Resat H, Borowski E. Comparative molecular dynamics simulations of amphotericin B-cholesterol/ergosterol membrane channels. Biochim. Biophys. Acta, 1567, 63-78 (2002).

26) Izumikawa K, Kohno S. Current status of drug delivery systems for antifungal drugs. Drug Delivery System, 27, 93-105 (2012).

27) Cybulska B, Gadomska I, Mazerski J, Borowski JGE, Cheron M, Bolard J. $N$-Methyl- $N$-D-fructosyl amphotericin B methyl ester (MFAME), a novel antifungal agent of low toxicity: monomer/micelle control over selective toxicity. Acta Biochim. Pol., 47, 121-131 (2000).

28) Sedlak M, Buchta V, Kubicova L, Simunek P, Holcapek M, Kasparova P. Synthesis and characterization of a new amphotericin Bmethoxy ployethylene glycol conjugate. Bioorg. Med. Chem. Lett., 11, 2833-2835 (2001).

29) Mozsolits H, Wirth H-J, Werkmeister J, Aguilar MI. Analysis of antimicrobial peptide interactions with hybrid bilayer membrane systems using surface plasmon resonance. Biochim. Biophys. Acta, 1512, 64-76 (2001)

30) Hiemenz JW, Walsh TJ. Lipid formulations of amphotericin B: recent progress and future directions. Clin. Infect. Dis., 22 (Suppl. 2), S133-S144 (1996). 